\title{
Temporal phase-unwrapping algorithm for dynamic interference pattern analysis in interference-contrast microscopy
}

\author{
Lennert R. van den Doel and Lucas J. van Vliet
}

\begin{abstract}
A temporal phase-unwrapping algorithm has been developed for the analysis of dynamic interference patterns generated with interference-contrast microscopy in micromachined picoliter vials. These vials are etched in silicon dioxide, have a typical depth of $6 \mu \mathrm{m}$, and are filled with a liquid sample. In this kind of microscopy, fringe patterns are observed at the air-liquid interface. These fringe patterns are caused by interference between the directly reflected part of an incident plane wave and the part of that wave that is reflected on the bottom of the vial. The optical path difference (OPD) between both parts is proportional to the distance to the reflecting bottom of the vial. Evaporation decreases the OPD at the meniscus level and causes alternating constructive and destructive interference of the incident light, resulting in an interferogram. Imaging of the space-varying OPD yields a fringe pattern in which the isophotes correspond to isoheight curves of the meniscus. When the bottom is flat, the interference pattern allows for monitoring of the meniscus as a function of time during evaporation. However, when there are objects on the bottom of the vial, the heights of these objects are observed as phase jumps in the fringes proportional to their heights. First, we present a classical electromagnetic description of interference-contrast microscopy. Second, a temporal phase-unwrapping algorithm is described that retrieves the meniscus profile from the interference pattern. Finally, this algorithm is applied to measure height differences of objects on the bottom in other micromachined vials with a precision of $\sim 5$ nm. (C) 2001 Optical Society of America

OCIS codes: $100.2650,120.2830$.
\end{abstract}

\section{Introduction}

Miniaturization for high-throughput screening applications was one of the most widely exploited fields of research during the past decade. In the future this technology will lead to a miniaturized lab-on-a-chip. Within the framework of an interfaculty research program, micromachined picoliter vials are fabricated in silicon dioxide with sizes ranging from 75 $\mu \mathrm{m} \times 75 \mu \mathrm{m}$ to $300 \mu \mathrm{m} \times 300 \mu \mathrm{m}$ and a typical depth of $6.0 \mu \mathrm{m}$. Each of these vials is equipped with a dedicated volume sensor. ${ }^{1}$ To calibrate this electronic sensor, optical monitoring of the evaporation

The authors are with the Pattern Recognition Group, Faculty of Applied Sciences, Delft University of Technology, Lorentzweg 1, NL-2628 CJ Delft, The Netherlands. L. R. van den Doel's e-mail address is L.R.vandenDoel.@ph.tn.tudelft.nl. L. J. van Vliet's e-mail address is L.J.vanVliet@ph.tn.tudelft.nl.

Received 6 July 2000; revised manuscript received 25 January 2001.

0003-6935/01/254487-14\$15.00/0

(C) 2001 Optical Society of America process in these subnanoliter vials is necessary. This requires a type of microscopy that allows for reconstruction of the shape of the liquid volume, especially from the air-liquid interface, during evaporation. This implies that the three-dimensional volume needs to be properly sampled in space (both lateral and axial) as well as in time.

Consider the case in which a vial is filled with a homogeneous fluorescent solution. The fluorescent signal of the particles indicates the presence of the liquid. With a conventional widefield microscope, however, a three-dimensional volume is imaged onto a two-dimensional image sensor after which depth information is lost. It is not straightforward to retrieve the three-dimensional shape of the liquid from these intensity differences.

A second well-established method to analyze threedimensional microvolumes is confocal fluorescence microscopy. This kind of microscopy, however, complies only with the first requirement. Because of the time-consuming scanning, the temporal resolution of confocal microscopy is poor.

A third type of microscopy that complies with both 

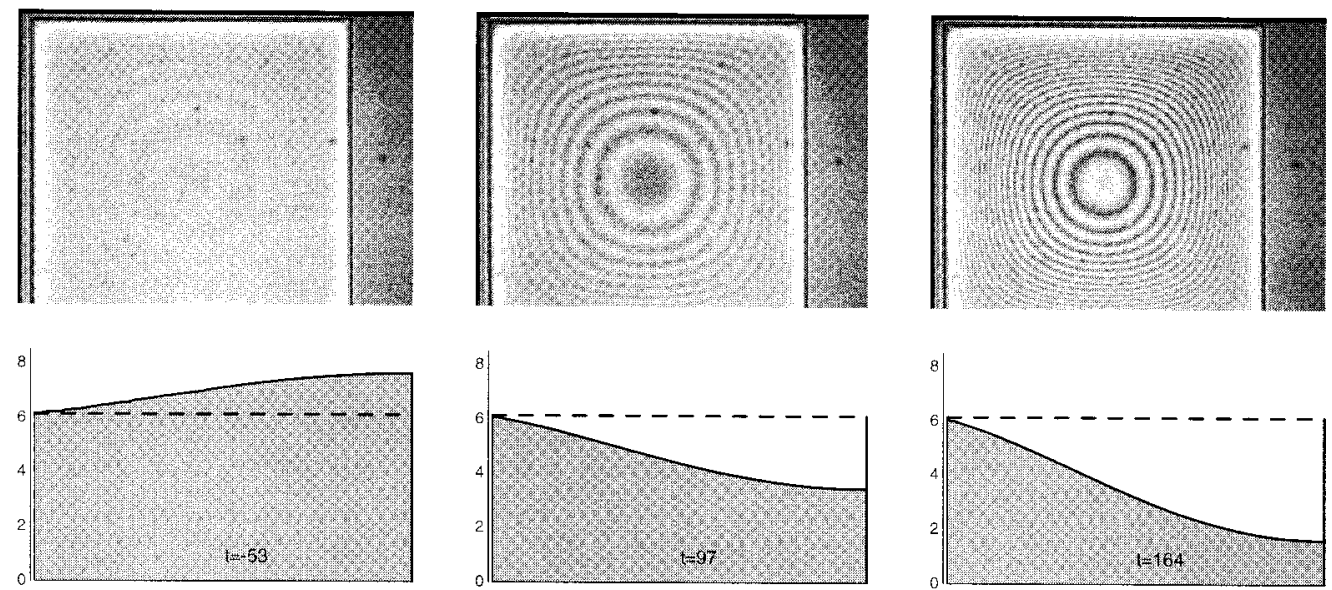

$t=-53 \mathrm{~s}$

$t=164 \mathrm{~s}$

$t=97 \mathrm{~s}$

Fig. 1. Top, dynamic interference patterns as recorded in a 6.0- $\mu \mathrm{m}$-deep vial. Bottom, one-dimensional height profiles of the meniscus along a diagonal from the corner to the center of the vial.

requirements is interference-contrast microscopy. The evaporation process of the liquid is observed with epi-illuminated imaging. Part of the incident light is reflected at the air-liquid interface. Another part is reflected at the bottom of the vial and interferes with the first reflected part at the air-liquid interface. This results in constructive and destructive interference as a function of the distance to the bottom of the vial. Evaporation of the liquid changes the profile of the meniscus. As a result, the interference patterns are dynamic. Imaging of the meniscus profile yields a fringe pattern in which the isophotes correspond to isoheight curves of the meniscus. Figure 1 shows three images of an acquired image sequence of the evaporation process in one of the vials. The instant $t=0 \mathrm{~s}$ corresponds to an image with a perfectly flat meniscus. Later on in this paper we discuss how this instant is measured. Furthermore Fig. 1 shows the height profiles of the meniscus, which resulted in the observed interferograms. These profiles are computed with a temporal phase-unwrapping algorithm presented here.

The following observations are made from the interferogram sequence acquired during evaporation of the liquid:

1. First, the meniscus is convex, and the fringes propagate toward the center of the vial and disappear. This indicates that the meniscus becomes flat. After the final fringe has disappeared, i.e., when the meniscus is perfectly flat, the fringes reappear in the center of the vial and propagate toward the sidewalls of the vial. During evaporation more and more fringes are generated. This implies that the meniscus becomes more concave. This is shown in the rightmost part of Fig. 1.

2. Starting from the instant when the meniscus is flat, and no fringes are present, until the moment that the liquid reaches the bottom and the liquid film breaks, 29 bright fringes appear in the center of the vial.

3. Just before the liquid breaks at the bottom of the vial, a dark fringe originates in the center of the vial.

4. The contrast between the dark and the bright fringes increases in time; i.e., the contrast increases with decreasing height. This can be seen in Fig. 1.

5. The spatial frequency of the fringes increases during evaporation. At a certain moment this results in spatial undersampling. Spatial phaseunwrapping algorithms will not be successful in these regions, owing to aliasing. As discussed below, our temporal phase-unwrapping algorithm will unwrap most of these regions successfully.

These observations can also be seen in Fig. 2. This figure shows the time evolution of the fringe pattern along a line through the center of the vial.

\section{Theory of Interference-Contrast Microscopy}

The optical model of interference-contrast microscopy is based on the classical theory of the generation of fringes of equal thickness in thin films. ${ }^{2}$ According to this theory, interference patterns are observed as a result of an optical path difference (OPD) between two parts of an incident plane wave. One part of the incident plane wave is reflected from the upper surface of the thin film, whereas the other part is reflected from the lower surface of the thin film. Lambacher and Fromherz ${ }^{3}$ expanded this model to describe microscopic observations of interference patterns generated by a fluorescent cyanine dye embedded in a Langmuir-Blodgett film. The dye is spaced from a reflecting silicon surface by a layer of silicon dioxide. Their optical model takes the distancedependent excitation of the fluorescent dye as well as the distance-dependent emission of the dye into account. Lambacher and Fromherz ${ }^{3}$ call this type of microscopy fluorescence interference-contrast mi- 


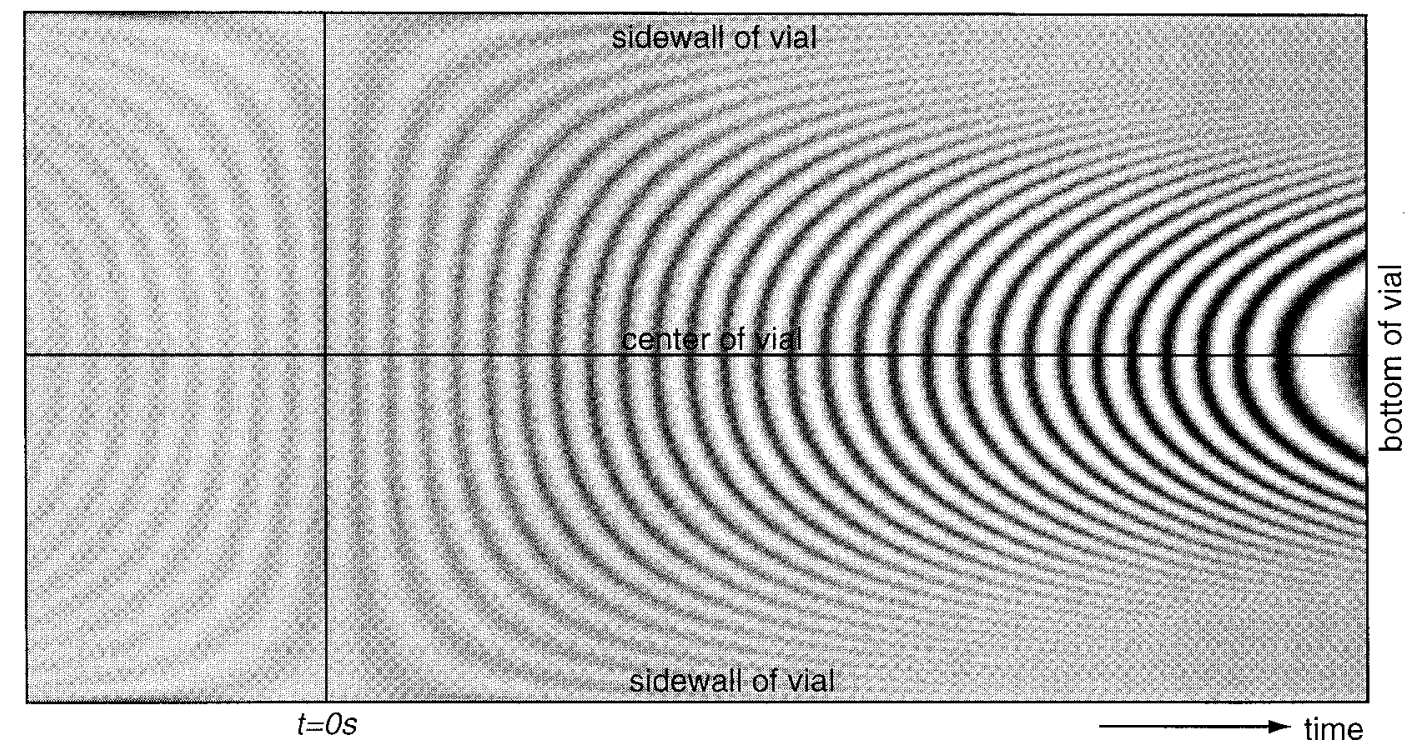

Fig. 2. Time evolution of the fringes along a line through the center of the vial. In the center of the vial most modulations occur, whereas along the sidewalls of the vial no modulations occur.

croscopy. In this paper we present an optical model to describe our observations of interference patterns in subnanoliter vials etched in silicon dioxide with a typical depth of $6 \mu \mathrm{m}$.

The observed interference patterns show modulations of the intensity of the electric field as a function of the liquid height $d$ (the meniscus) in the vial. The optical model of interference-contrast microscopy is shown in Fig. 3. The microscope system is adjusted to Köhler illumination ${ }^{4}$ : This implies incoherent illumination, and the incident light consists of plane waves. After refraction at the air-liquid interface, the plane wave propagates toward the reflecting bottom of the vial. The direct part of the incident plane wave interferes with the part of the incident plane wave that is reflected at the bottom of the vial.

The OPD between the direct part and the reflected

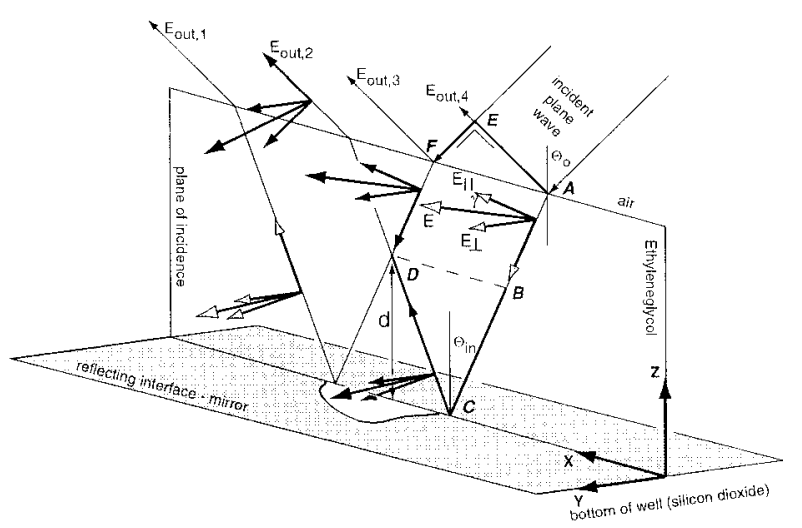

Fig. 3. Incident plane wave refracted at the air-liquid interface of the liquid sample in the vial. After refraction the direct part of this plane wave interferes with the part of this plane wave that is reflected on the bottom of the vial. Whether this interference is constructive or destructive depends on the height $d$ with respect to the bottom of the vial. part of the incident plane wave at a height $d$ above the reflecting bottom of the vial is given by

$$
\mathrm{OPD}=2 n_{\text {liq }} d \cos \left(\Theta_{\text {in }}\right),
$$

where $n_{\text {liq }}$ is the refractive index of the liquid (ethylene glycol, $\left.n_{\text {lig }}=1.432\right)$ and $\Theta_{\text {in }}$ is the angle of incidence in the liquid sample.

This OPD causes interference between both parts of the plane wave as a function of the height $d$ above the bottom of the vial. The modulations at a height $d$ in the liquid, however, cannot be observed. The reason for this is that in a microscope system the outcoming rays, indicated in Fig. 3 as $E_{\text {out }, i}$, are observed in different uncorrelated points on an image sensor. An image sensor mounted on a microscope can observe interference only if two rays combine in a single point on the image sensor, which seem to originate from the same point in the object plane but have different optical paths. According to Fig. 3 the rays $E_{\text {out }, 2}$ and $E_{\text {out, } 3}$ have different optical paths but do not originate from the same point from an image sensor point of view. When $d$ is extended to the air-liquid interface, however, these two rays shift toward each other and end up in the same position. The image sensor mounted on the microscope will observe this as interference. This implies that our observed interference patterns originate at the airliquid interface and that the modulations inside the liquid cannot be observed at all.

The phase difference $\Phi_{\text {in }}$ corresponding to the OPD given by Eq. (1) is

$$
\Phi_{\text {in }}=\frac{4 \pi n_{\text {liq }} d \cos \left(\Theta_{\mathrm{in}}\right)}{\lambda},
$$

where $\lambda$ is the wavelength of the incident light.

As indicated in Fig. 3, the electric field vectors of the incident plane wave are split into vectors normal 
to the plane of incidence $\left(\mathbf{E}_{\perp}\right)$ and vectors parallel to the plane of incidence $\left(\mathbf{E}_{\|}\right)$. With the notation from Fig. 3 the electric field vector of the incident plane wave $\mathbf{E}_{i}$ and the electric field vector $\mathbf{E}_{r}$ after reflection at the air-liquid interface are given in Cartesian coordinates by

$$
\begin{aligned}
& \mathbf{E}_{i}=E\left[\begin{array}{c}
\cos \left(\gamma_{0}\right) \cos \left(\Theta_{0}\right) \\
\sin \left(\gamma_{0}\right) \\
\cos \left(\gamma_{0}\right) \sin \left(\Theta_{0}\right)
\end{array}\right], \\
& \mathbf{E}_{r}=E\left[\begin{array}{c}
\cos \left(\gamma_{0}\right) \cos \left(\Theta_{0}\right) r_{01} \\
\sin \left(\gamma_{0}\right) r_{01}^{\perp} \\
\cos \left(\gamma_{0}\right) \sin \left(\Theta_{0}\right) r_{01}^{\|}
\end{array}\right],
\end{aligned}
$$

where $E$ is the amplitude of the electric field of the incident plane wave. The angle $\gamma_{0}$ is the angle of polarization with respect to the plane of incidence. The angle $\Theta_{0}$ is the angle of incidence of the incident plane wave. The coefficients $r_{i j}^{\|}$and $r_{i j}^{\perp}$ are the Fresnel coefficients for reflection at the interface between media $i$ and $j$ for the parallel component and the normal component, respectively. The index 0 is for the air, 1 for the liquid in the vial, and 2 for the silicon dioxide under the bottom of the vial. The electric field vector $\mathbf{E}_{\text {trt }}$ after refraction at the airliquid interface, reflection at the bottom of the vial, and again refraction at the liquid-air interface is given in Cartesian coordinates by

$$
\mathbf{E}_{\text {trt }}=E \exp \left(i \Phi_{\text {in }}\right)\left[\begin{array}{c}
\cos \left(\gamma_{0}\right) \cos \left(\Theta_{0}\right) t_{01}^{\|} r_{12}^{\|} t_{10}^{\|} \\
\sin \left(\gamma_{0}\right) t_{01}^{\perp} r_{12}^{\perp} t_{10}^{\perp} \\
\cos \left(\gamma_{0}\right) \sin \left(\Theta_{0}\right) t_{01}^{\|} r_{12}^{\|} t_{10}^{\|}
\end{array}\right],
$$

where $\Phi_{\text {in }}$ is the phase difference as defined in Eq. (2). The coefficients $t_{i j}^{\|}$and $t_{i j}^{\perp}$ are the Fresnel coefficients for transmission through the interface between media $i$ and $j$ for the parallel component and the normal component, respectively.

The sum of the two parts of the reflected plane waves follows then as

$$
\mathbf{E}=E\left[\begin{array}{c}
\cos \left(\gamma_{0}\right) \cos \left(\Theta_{0}\right)\left[r_{01}^{\|}+t_{01}^{\|} r_{12}^{\|} t_{10}^{\|} \exp \left(i \Phi_{\text {in }}\right)\right] \\
\sin \left(\gamma_{0}\right)\left[r_{01}^{\perp}+t_{01}^{\perp} r_{12}^{\perp} t_{10}^{\perp} \exp \left(i \Phi_{\text {in }}\right)\right] \\
\cos \left(\gamma_{0}\right) \sin \left(\Theta_{0}\right)\left[r_{01}^{\|}+t_{01}^{\|} r_{12}^{\|} t_{10}^{\|} \exp \left(i \Phi_{\text {in }}\right)\right]
\end{array}\right]
$$

The intensity of the electric field describes the observed interference as a function of the height $d$ for a single incident plane wave with a certain angle of polarization, angle of incidence, and wavelength. The sum of all these single-quantum modulations gives rise to the observed interference patterns. ${ }^{3}$ The first step to compute the intensity of the electric field is to integrate $|\mathbf{E}|^{2}$ analytically over all angles of polarization. The result of this computation can be split into an offset, which will not be taken further into account, and a modulating part:

$$
\begin{aligned}
\left\langle|E|^{2}\right\rangle_{\gamma_{0}}= & \pi\left(r_{01}^{\perp 2}+r_{01}^{\| 2}+t_{01}^{\perp 2} r_{12}^{\perp 2} t_{10}^{\perp 2}+t_{01}^{\| 2} r_{12}^{\| 2} t_{10}^{\| 2}\right) \\
& +2 \pi\left(r_{01}^{\perp} t_{01}^{\perp} r_{12}^{\perp} t_{10}^{\perp}+r_{01}^{\|} t_{01}^{\|} r_{12}^{\|} t_{10}^{\|}\right) \cos \left(\Phi_{\text {in }}\right) .
\end{aligned}
$$

The amplitude term preceding the cosine function shows little dependence on the angle of incidence $\Theta_{\text {in }}$ in the interval of interest [0,0.2] rad.

The second step to evaluate the intensity of the electric field is performed by means of averaging over all wavelengths. For the experiments a narrowbandpass filter with a central wavelength $\lambda_{c}=602.3$ $\mathrm{nm}$ and a FWHM of $9.7 \mathrm{~nm}$ is placed in the illumination path. The transmission spectrum $S(\lambda)$ (normalized in amplitude) of this filter can be approximated by a flattened Gaussian function,

$$
S(\lambda)=\left[1+\frac{\left(\lambda-\lambda_{c}\right)^{2}}{2 \sigma_{\lambda}^{2}}\right] \exp \left[-\frac{\left(\lambda-\lambda_{c}\right)^{2}}{2 \sigma_{\lambda}^{2}}\right],
$$

with $\sigma_{\lambda}=2.56 \mathrm{~nm}$ corresponding to the FWHM of the filter.

Finally, we introduce a weight function for the angle of incidence. The microscope system used for the experiments is adjusted to Köhler illumination. This implies that the back focal plane of the objective is a congruent plane of the light source. Furthermore, we assume that the back focal plane is uniformly filled. Each point in the back focal plane corresponds to a certain angle of incidence $\Theta^{\prime}$, according to the relation $r^{\prime} \propto \tan \left(\Theta^{\prime}\right)$, with $r^{\prime}$ the distance to the optical axis in the back focal plane and $\Theta^{\prime}$ the angle of incidence. All points with the same distance to the optical axis correspond to the same angle of incidence. The probability that an incident plane wave has an angle of incidence smaller than the angle $\Theta^{\prime}$ is proportional to $\pi r^{\prime 2}$, which is a circular fraction of the back focal plane. The probability that the angle of incidence of a plane wave is smaller than the maximum angle of incidence $\Theta_{\text {max }}$ is, of course, 1 , proportional to $\pi R^{2}$, with $R$ the radius of the back focal plane. The cumulative probability density function then equals $\left(r^{\prime} / R\right)^{2}$. The probability density function that a certain incident plane wave has an angle of incidence $\Theta^{\prime}$ is then equal to $2 r^{\prime} / R^{2}$, which is proportional to $\tan \left(\Theta^{\prime}\right)$. This defines the weight function for the different angles of incidence.

The halogen lamp used for the experiments, however, does not fill the back focal plane of the objective (Zeiss FLUAR $20 \times / 0.75$ ) completely. Given the definition of the numerical aperture $\left[\mathrm{NA}=n_{\text {air }}\right.$ $\left.\sin \left(\Theta_{0, \max }\right)\right]$ and a light source large enough to fill the back focal plane, the following relation yields for the maximum angle of incidence

$$
\tan \left(\Theta_{0, \text { max }}\right)=\frac{\mathrm{NA}}{\sqrt{n_{\text {air }}^{2}-\mathrm{NA}^{2}}} .
$$

The halogen lamp fills only one fifth the back focal plane. In that case, $\tan \left(\Theta_{0, \max }\right)$ equals one fifth the right-hand side of Eq. (9). The angles of incidence $\Theta_{0}$ and $\Theta_{\text {in }}$ are related by Snellius's law. The max- 


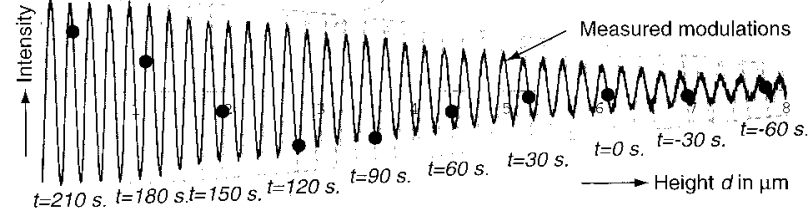

Fig. 4. Intensity of the electric field as a function of the height $d$ above the reflecting bottom of the vial. One curve is based on evaluation of Eq. (11), whereas the other curve is experimentally acquired. The moment $t=0 \mathrm{~s}$ corresponds to the moment in the recording of the interference pattern where the meniscus is perfectly flat.

imum angle of incidence in the liquid $\Theta_{\text {in,max }}$ follows then as

$$
\Theta_{\mathrm{in}, \max }=\arcsin \left\{\frac{n_{\text {air }}}{n_{\mathrm{liq}}} \sin \left[\arctan \left(\frac{1}{5} \frac{\mathrm{NA}}{\sqrt{n_{\mathrm{air}}^{2}-\mathrm{NA}^{2}}}\right)\right]\right\} .
$$

With these two weight functions for the wavelength and the angle of incidence of the incident light, the intensity of the electric field at a height $d$ above the bottom of the vial can be computed as

$$
|\mathbf{E}(d)|^{2}=\int_{\Theta_{\mathrm{in}}=0}^{\Theta_{\mathrm{in}, \max }} \int_{\lambda=0}^{\infty} \tan \left(\Theta_{\mathrm{in}}\right) S(\lambda)\left\langle|\mathbf{E}|^{2}\right\rangle_{\gamma_{0}} \mathrm{~d} \Theta_{\mathrm{in}} \mathrm{d} \lambda,
$$

where $S(\lambda)$ is defined in Eq. (8) and $\left\langle|\mathbf{E}|^{2}\right\rangle_{\gamma_{0}}$ in Eq. (7). The maximum angle of incidence $\Theta_{\text {in,max }}$ follows from Eq. (10).

Equation (11) is evaluated numerically as a function of the height $d$ above the bottom of the vial with $\lambda_{c}=602.3 \mathrm{~nm}, \sigma_{\lambda}=2.56 \mathrm{~nm}, n_{\text {liq }}=1.4319, n_{\text {sil }}=$ 1.46 , and $\Theta_{\max }=0.155 \mathrm{rad}$. The result of this computation is shown in Fig. 4. The bottom of the vial is covered by a layer of uniform thickness of $\mathrm{Si}_{x} \mathrm{~N}_{y}$. The incident light is reflected at the $\mathrm{Si}_{x} \mathrm{~N}_{y}-\mathrm{SiO}_{2}$ interface. The refractive index of $\mathrm{Si}_{x} \mathrm{~N}_{y}$ is approximately 2.1. This implies that no phase shift of $\pi \mathrm{rad}$ occurs at reflection on this interface. Of course, this layer introduces an additional constant OPD. This extra OPD almost equals an integer number of periods for the wavelengths used in the experiments. Therefore it is not taken into account, and we subtract a phase shift of $\pi$ rad from the simulation results to remove the extra phase shift introduced by reflection on the liquid- $\mathrm{SiO}_{2}$ interface used in the model. This computation is compared with measurements in a digital recording of an evaporating ethylene glycol sample. In each frame of this recording the intensity in the center of the vial is measured. The modulating intensity, measured as a function of time, is unwrapped with our temporal phaseunwrapping algorithm presented in Section 3. This temporal phase-unwrapping algorithm gives the height of the liquid as a function of time. A parametric plot of the modulating intensity against the height of the liquid is also shown in Fig. 4. Recall our observations mentioned in Section 1. Our second observation was that 29 bright fringes appeared in the center of the vial from the moment that the meniscus was perfectly flat $(t=0 \mathrm{~s})$. This can be checked in Fig. 4. Our third observation was that the final fringe just above the bottom of the vial is a dark fringe. Our model complies with this. Furthermore, our fourth observation was that the contrast increases with decreasing height. This can also be seen in Fig. 4. Finally, as can be seen in Fig. 4 , the length of the measured modulations equals the length of the simulated modulations from Eq. (11). The amplitude of the measured modulations, however, drops much faster than that of the simulated modulations. We believe that the poor agreement between our model and the experiments is due to out-of-focus light. The focal plane for the experiments is the bottom of the vial. This implies that in the beginning of the recording, when the meniscus is convex as shown in Fig. 1, the meniscus is not focused and contains large out-of-focus contributions. During the evaporation the meniscus becomes increasingly in focus, and the contributions of the outof-focus light become smaller and finally disappear. The contributions of the out-of-focus light result in a reduced contrast for large values of $d$.

The simulation results in Fig. 4 indicate that the modulations can be written as $A(d) \cos [\Phi(d)]$, where $\Phi(d)$ is a function that defines the length of the modulations and $A(d)$ is a function that defines the envelope of the modulations. The argument of the cosine function $\Phi(d)$ is, of course, related to the phase difference as defined in Eq. (2). The different wavelengths are replaced with a single effective wavelength $\lambda^{\text {eff }}$, and the different angles of incidence are replaced with a single effective angle of incidence $\Theta_{\mathrm{in}}^{\text {eff }}$ This yields

$$
\Phi(d)=\frac{4 \pi n_{\text {liq }} d \cos \left(\Theta_{\text {in }}^{\mathrm{eff}}\right)}{\lambda^{\mathrm{eff}}},
$$

From this equation it follows that the length of the modulations $\Delta d$, i.e., the change in the height $d$ corresponding to a phase difference $\Phi(d)=2 \pi$, equals

$$
\Delta d=\frac{\lambda^{\text {eff }}}{2 n_{\text {liq }} \cos \left(\Theta_{\text {in }}^{\text {eff }}\right)} .
$$

Because of the symmetry of the transmission spectrum $S(\lambda)$ around $\lambda=\lambda_{c}$, we conclude that the length of the modulations is proportional to $\lambda_{c}$. This implies that the effective wavelength $\lambda^{\text {eff }^{c}}=\lambda_{c}$. The effective angle of incidence $\Theta_{\text {in }}^{\text {eff }}$ corresponds to the effective radius of the back focal plane $R / \sqrt{2}$; thus $\Theta_{\text {in }}^{\text {eff }}=\Theta_{\text {in,max }} / \sqrt{2}$.

For small values of $d$ the length of the modulations depends weakly on the angle of incidence. ${ }^{2}$ For large values of $d$, however, both the angular distribution and the spectral bandwidth will have a significant effect on the fringe visibility. Finally, we compared the positions of the zero crossings in our measured modulations with the predicted positions of 
the zero crossings $(2 k+1) / 4 \times \Delta d, k \in\{0,1,2$, $3, \ldots\}$. The error between the measured positions of the zero crossings and the predicted positions has a standard deviation of $2.5 \mathrm{~nm}$, which equals approximately $1 \%$ of the length of the modulations.

The envelope or amplitude factor $A(d)$ of the modulations is given by the Fourier transform of the transmission spectrum: In a manner similar to that in a Fourier-transform spectrometer ${ }^{5}$ the produced interogram encodes the spectrum of the light. Note that the transmission spectrum $S(\lambda)=S\left(\lambda, \sigma_{\lambda}\right)$ is characterized by its central wavelength $\lambda_{c}$ and width $\sigma_{\lambda}$. This implies that the transmission spectrum $S\left(k, \sigma_{k}\right)$ in terms of the wave number $k$ and the width $\sigma_{k}$ has the same normalized standard deviation, i.e., $\sigma_{k} / k_{c}=\sigma_{\lambda} / \lambda_{c}$, with $k_{c}=2 \pi / \lambda_{c}$. The envelope $A(d)$ is given by, neglecting constant factors,

$$
\begin{aligned}
A(d)= & \int_{\Theta_{\mathrm{in}}=0}^{\Theta_{\mathrm{in}, \max }} F\left[\Theta_{\mathrm{in}}^{\mathrm{eff}}\right] \tan \left(\Theta_{\mathrm{in}}^{\mathrm{eff}}\right) \\
& \times \sigma_{k}\left[4 n_{\text {liq }}^{2} d^{2} \sigma_{k}^{2} \cos ^{2}\left(\Theta_{\mathrm{in}}^{\mathrm{eff}}\right)-3\right] \\
& \times \exp \left[-2 \sqrt{3} n_{\text {liq }}^{2} d^{2} \sigma_{k}^{2} \cos ^{2}\left(\Theta_{\mathrm{in}}^{\text {eff }}\right)\right] \mathrm{d} \Theta_{\mathrm{in}},
\end{aligned}
$$

where $F[\cdot]$ stands for the amplitude term of all Fresnel coefficients in Eq. (7). An extra factor of $\sqrt{3}$ is added to the exponential term for proper scaling.

\section{Phase Unwrapping: Spatial versus Temporal}

The acquired interferograms $I[m, n ; t]$ as shown in Figs. 1 and 2 can be described by

$$
\begin{aligned}
I[m, n ; t]= & A[m, n ; t] \cos (\Phi[m, n ; t])+B[m, n ; t] \\
& +N[m, n ; t],
\end{aligned}
$$

where $A[\cdot]$ and $B[\cdot]$ are the space-time varying amplitude and background. The argument $\Phi[m, n$; $t]$ is the phase map of the interferogram. The phase map is related to the height of the liquid by means of Eq. (12). The signal $N[m, n ; t]$ is an additive noise signal. The goal of an unwrapping algorithm is first to derive the wrapped phase map $\phi[m, n ; t]$ from the observed interferogram $I[m, n ; t]$ by means of measuring the relative phase at every time. The second step is to retrieve the absolute phase map $\Phi[m, n ; t]$ with respect to a reference point by addition of $2 \pi$ to the relative phase at every located $2 \pi$ discontinuity. Below we describe how this reference point is determined.

Several algorithms exists that unwrap twodimensional phase maps derived from a static interference pattern, e.g., Refs. 6 and 7. These algorithms compare phase values at neighboring pixels. Spatial unwrapping algorithms might experience the following three problems. First, phase errors originating in regions with a high noise level propagate outside these regions and corrupt the remaining part of the image. Second, the fringe pattern must be sampled at a spatial frequency at least as high as twice the highest frequency in the interferogram to meet the requirements of the Nyquist sampling theorem. In
Section 1 we observed that the images are undersampled in regions with a steep meniscus. In this section we treat the aspects of sampling in detail. A third problem of spatial unwrapping algorithms involves true discontinuities in the phase map. These discontinuities are observed as segmented fringes and are caused by height differences. Experimental results prove that our temporal phaseunwrapping algorithm can easily deal with these discontinuities.

To avoid the problems mentioned above, we propose to analyze the acquired interferograms in time, point by point. Temporal phase unwrapping was described earlier, e.g., by Huntley and Saldner. ${ }^{8}$ Huntley and Saldner use a four-step interferometer to generate four intensity images at phase steps of $\pi / 2$. A phase map is directly computed from these four images. With this method the phase difference between two successive phase maps is straightforward to compute. However, in our case, the interferograms are not recorded with well-known phase steps between two successive images. On the contrary, the phase difference between two successive images measured at different positions is a continuum that is due to evaporation of liquid during the experiments. As can be seen in Fig. 1 and other figures in this paper, the total height change in the center of the vial is maximal. This leads to a maximal phase difference at that position in two successive images. However, at the border of the vial there is practically no height change: The phase difference is minimal.

\section{A. Temporal Phase-Unwrapping Algorithm}

In the remainder of this section the spatial dependency of the physical quantities in Eq. (15) will be dropped: $\quad I[t]=I[m, n ; t]$ and so forth. The square brackets indicate that the signals are discrete signals. Figure 5 shows the successive steps of the temporal phase-unwrapping algorithm.

The first step in the computation of the phase map $\Phi[t]$ from the interferogram $I[t]$ is to subtract the background $B[t]$ from the interferogram. The timedependent background is estimated by low-pass filtering $I[t]$ with a Gaussian kernel with a large standard deviation. The result of this operation is denoted as $I^{\prime}[t]$. The signal $I^{\prime}[t]$ consists of the cosine function with varying amplitude and the additive noise term.

The next step is to subsample the low-frequency signal $I^{\prime}[t]$ with a factor $f_{\text {sub }}$. The temporal sampling density is constant, but the temporal bandwidth varies spatially as can be seen in Fig. 2 . To satisfy the Nyquist criterion in the time dimension over the entire image, the signal in time is oversampled: The oversampling rate increases toward the sidewalls of the vial. Furthermore, we know that a window function is required for proper phase estimation. For these reasons we propose using a window function with a fixed width of 128 points and subsampling the low-frequency signal $I^{\prime}[t]$. This subsampling is performed such that approximately 


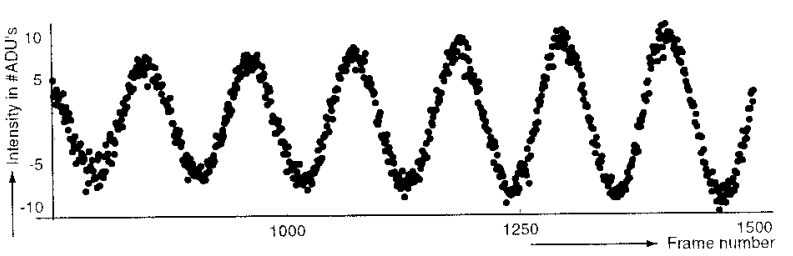

(a)

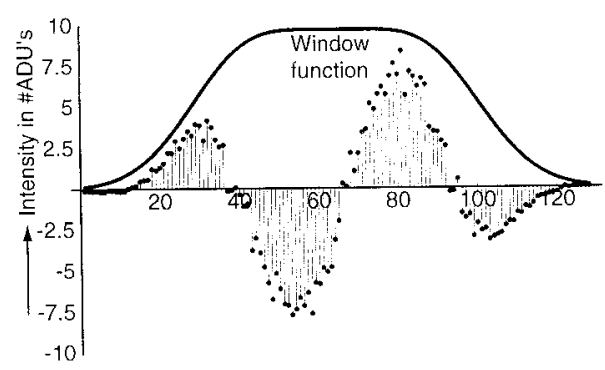

(b)

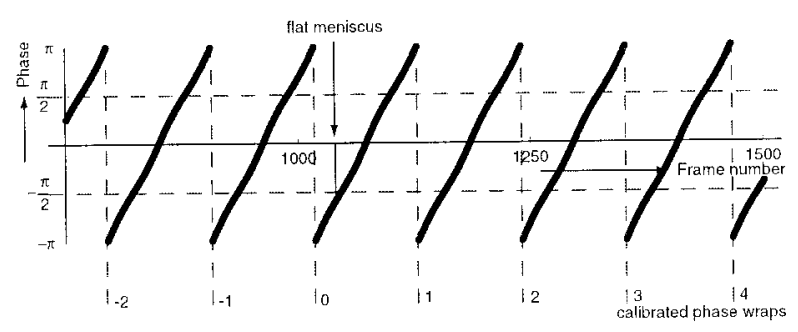

(c)

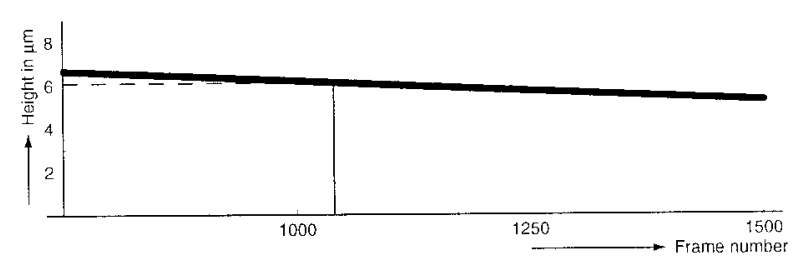

(d)

Fig. 5. Temporal phase-unwrapping algorithm: (a) backgroundcorrected signal $I^{\prime}[t]$ in the center point of the vial in the frame series 750-1500, (b) window function $W[t]$ and the product $I_{\text {sub }}^{\prime}[t] W[t]$, (c) estimated phases, (d) the height profile, which follows by unwrapping of the estimated wrapped phases.

two periods of $I_{\text {sub }}^{\prime}[t]$ fall within the width of a window $W[t]$. The window function is defined by

$$
\begin{aligned}
W[t]= & {\left[1+\frac{1}{2}\left(\frac{t-K_{w} / 2}{\sigma_{t}}\right)^{2}+\frac{1}{8}\left(\frac{t-K_{w} / 2}{\sigma_{t}}\right)^{4}\right] } \\
& \times \exp \left[-\frac{\left(t-K_{w} / 2\right)^{2}}{2 \sigma_{t}^{2}}\right],
\end{aligned}
$$

where $\sigma_{t}=16.0$ and $K_{w}$ is the fixed width of the window. The subsampling factor $f_{\text {sub }}$ is defined as the ratio between the average length of the modulations in $I^{\prime}[t]$ and the required average length of the modulations within the width $K_{w}$ of the window. The former equals the number of data points $N$ in $I^{\prime}[t]$ divided by the number of periods in $I^{\prime}[t]$, where each period has two zero crossings $Z C$, and the latter simply equals $K_{w} / 2$. Thus the subsampling factor $f_{\text {sub }}$ yields

$$
f_{\text {sub }}=\left[\frac{2}{K_{w}} \frac{2 N_{I^{\prime}[t]}}{\# Z C}\right],
$$

where $[\cdot]$ is the round operator.

To estimate the number of zero crossings $\# Z C$ in $I^{\prime}[t]$, we apply a low-pass filter in the time dimension with a variable width $\sigma$ that suppresses the noise sufficiently but does not corrupt the signal. This implies that the width of the filter must be tuned such that it removes all zero crossings introduced by the noise but not the zero crossings of the signal. First, the number of zero crossings \#ZC is counted in $I^{\prime}[t]$. Then $I^{\prime}[t]$ is filtered with a Gaussian kernel $G[t ; \sigma=$ $\sqrt{2}]$, and the number of zero crossings is counted again. This operation removes zero crossings that are due to the high-frequency components of the noise. This filtering is repeated with the size of $G[t$; $\sigma]$ increased by a factor of $\sqrt{2}$ until the number of zero crossings does not change in two successive filterings. In this situation the noise is suppressed sufficiently, and only true zero crossings are present in the signal. The number of zero crossings varies from 1 at the sidewall of the vial to 84 in the center of the vial. Under the assumption that the evaporation rate remains constant, the average number of periods within $K_{w}$ can be computed with Eq. (17). As a result of the resampling the average number of modulations within $K_{w}$ of the subsampled interferogram varies from 1.62 to 2.39 modulations. The subsampled signal is denoted as $I_{\text {sub }}^{\prime}[t]$. This method of subsampling is necessary to keep $\mathscr{F}\left\{I_{\text {sub }}^{\prime}[t] W[t]\right\}$ from mixing with the dc component of the frequency spectrum. The operator $\mathscr{F}\{\cdot\}$ denotes the Fourier transform.

Note that the discrete Fourier transform is a leastsquares optimization algorithm for a number of uniformly spaced frequencies. ${ }^{9}$ The aforementioned subsampling strategy ensures a proper match between the actual frequency and two times the fundamental frequency $2 \pi / N$ of the discrete frequencies of the discrete Fourier transform.

The third step in the algorithm is to compute the one-dimensional wrapped phase map $\hat{\Phi}_{\text {sub }}[t]$. One way to do this is to apply a fast-Fourier-transform algorithm to get a Fourier transform of a windowed part of $I_{\text {sub }}^{\prime}[t]$. The window function avoids discontinuities in the periodic representation of $I_{\text {sub }}^{\prime}[t] \mathrm{com}-$ puted with the discrete Fourier transform. The phase to be estimated equals the phase of the bin with the maximum amplitude in the discrete frequency spectrum. For each point $I_{\text {sub }}^{\prime}\left[t_{0}\right]$ the wrapped phase $\hat{\Phi}_{\text {sub }}\left[t_{0}\right]$ is computed by multiplication of a 128-point-wide window $W[t]$ with $I_{\text {sub }}^{\prime}\left[t+t_{0}\right]$ 


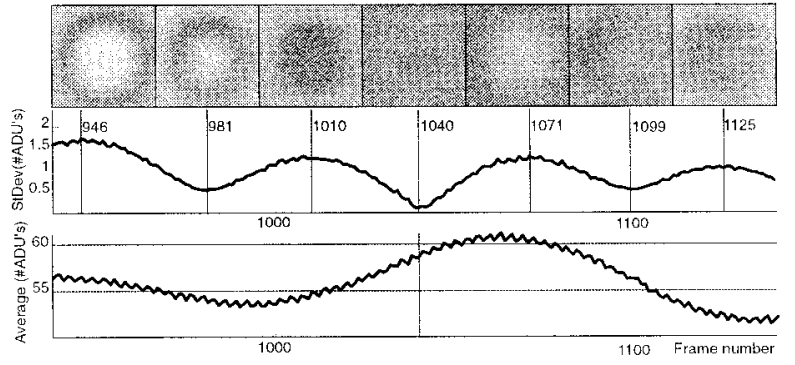

Fig. 6. The frame, where the meniscus is perfectly flat, is determined by a minimum variation in intensity. This frame is used to trigger all relative height profiles when the number of counted $2 \pi$ discontinuities is set to zero in this frame.

around the instant $t_{0}$ and computing the fast Fourier transform of this product. The wrapped phase at the instant $t_{0}$ follows from the discrete frequency spectrum: $\hat{\Phi}_{\text {sub }}\left[t_{0}\right]=\operatorname{Phase}\left\{W[t] I_{\text {sub }}^{\prime}\left[t_{0}\right]\right\}$.

After these three steps the one-dimensional wrapped phase map is computed. Next, the wrapped phase map is calibrated with respect to a reference point as described in Subsection 3.B. In this calibration step the number of observed phase wraps is set to zero at the instant where the meniscus is flat; i.e., we start counting phase wraps from this instant on. The final step of the algorithm is to unwrap the wrapped phase map. The unwrapping is done with an unwrapping operator found in the literature. ${ }^{7}$ Finally, the subsampled unwrapped phase map $\Phi_{\text {sub }}[t]$ is interpolated by a factor $f_{\text {sub }}$ to the original length of $I[t]$. The phase map $\Phi[t]$ is scaled with a factor $\Delta d / 2 \pi$ to an absolute height in micrometers. The result of this unwrapping algorithm is the height of the liquid $d[m, n ; t]$ in a single data point $[m, n]$ as a function of time.

\section{B. Computation of Reference Frame}

It is not possible to calibrate all wrapped phase maps at the final image of the image sequence, because the liquid does not reach the bottom of the vial at every point at the same time. The wrapped phase maps can be calibrated, however, when the meniscus is flat. At that instant an interference pattern is absent, because the meniscus is parallel to the bottom of the vial. In this time interval the OPD is approximately independent of the position. Although no fringe pattern is being observed, the measured intensity over the entire vial is influenced by the actual phase difference. This means that the intensity over the entire vial shows the same modulation in time, owing to the time-varying OPD when the meniscus changes from convex to concave. This was monitored and is shown in Fig. 6. The bottom graph shows the modulation of the average intensity as a function of time, whereas the top graph shows the standard deviation of the intensity (measured after background correction). The frame with the smallest variation in average intensity corresponds to the one with the flat

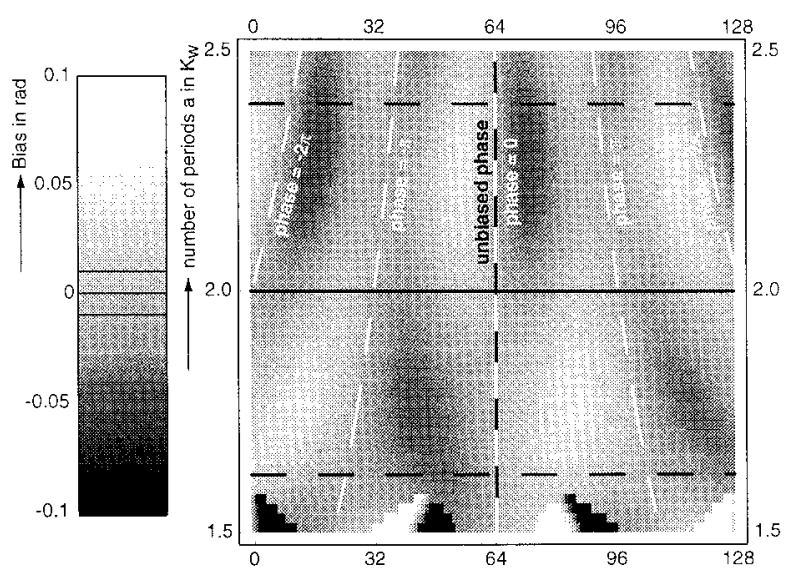

Fig. 7. Bias of the phase estimation for a cosine function with a varying number of periods $\alpha$ in $K_{w}$. The cosine function has unit amplitude and no noise. The bias is less than $0.01 \mathrm{rad}$. In the region below $\alpha=1.58$ the bias is at least 1 order of magnitude larger.

meniscus. In this figure the standard deviation of a frame $i$ is computed as

$$
\operatorname{StDev}\left(I_{i}[m, n]-\langle I[m, n]\rangle_{t=1010, \ldots, 1070}\right),
$$

where $\langle I[m, n]\rangle_{t}$ is the time average over 60 frames in the time window where the meniscus is almost flat. From Fig. 6 it follows that the meniscus becomes perfectly flat in frame 1040 . The time $t=0$ in Fig. 4 corresponds to this frame number. All wrapped phase maps are calibrated by means of setting the number of observed phase wraps at that instant to zero. The labels of the calibrated phase wraps are indicated in Fig. 5.

The instant of the flat meniscus defines the absolute height of the vial. The absolute height of the vial follows from Fig. 4: The height of the vial is 6.13 $\mu \mathrm{m}$.

\section{Precision and Accuracy of Phase Estimation}

The phase estimation is biased. Figure 7 shows the bias of the phase estimation of the temporal unwrapping algorithm. The bias of the phase estimate is computed by means of unwrapping $\cos (\Phi[t])$ with unit amplitude and no noise. The argument of the cosine function $\Phi[t]$ is given by $2 \alpha \pi / K_{w} \times\left[t-\left(K_{w} /\right.\right.$ $2)]$, where $\alpha$ defines the number of periods of $\Phi[t]$ within $K_{w}$. The number of periods varies from 1.5 periods at the bottom of this graph to 2.5 periods at the top. Recall that the number of periods within $K_{w}$ varies approximately from 1.6 to 2.4 periods, owing to the subsampling. The bias is less than $0.01 \mathrm{rad}$. It can be seen in Fig. 7 that the bias of phase estimation becomes large for windows smaller than 1.58 periods. The bias in these regions is at least 1 order of magnitude larger than in the remaining part of this figure. Varying the amplitude $A[t]$ results in a larger bias. The bias increases when the number of periods within $K_{w}$ deviates more from 2 . The bias of the phase estimation with exactly 2 periods within $K_{w}$ is minimal. The phase estimation $\Phi=0$ is unbiased 


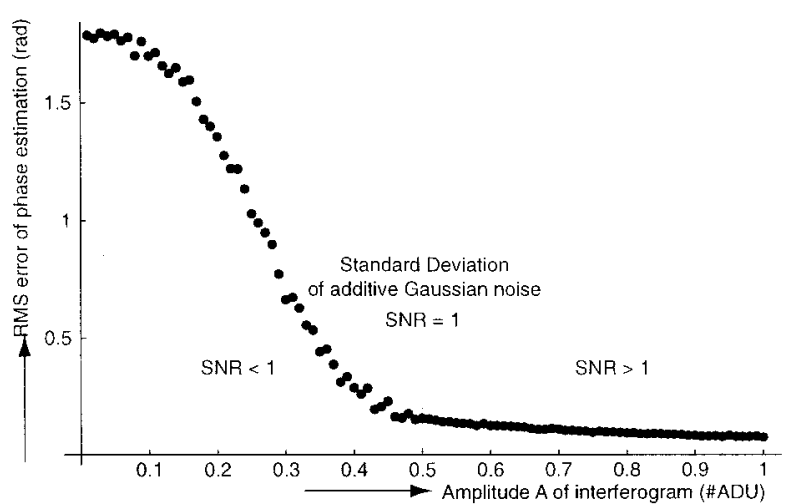

Fig. 8. RMS error of the phase estimation as a function of the amplitude of the interferogram with a constant noise level. Even when the amplitude equals the standard deviation of the noise, the phase can still be estimated with a RMS error of 0.16. If the SNR is below 1 , the RMS error increases rapidly.

only when the amplitude $A[t]$ is constant. The shape of the regions below 1.58 periods is maintained.

So far we have not taken the additive noise term of Eq. (15) into account. Figure 5 shows a part of the interferogram in the center of the vial. The amplitude $A[t]$ is of the order of $7 \mathrm{ADU}$ (anolog-to-digital units). The standard deviation of the noise $\sigma_{N}$ is estimated by

$$
\sigma_{N}=\operatorname{StDev}\{I[t]-I[t] * G[t ; \sigma=2.0]\},
$$

where $G[\cdot ; \sigma]$ is a Gaussian kernel with standard deviation $\sigma$ and $*$ is the convolution operator. A typical noise level for the measurements presented in this paper is $\sigma_{N}=0.77$. The signal-to-noise ratio (SNR) is of the order of 10 . We measure the phaseestimation variation by applying the algorithm to a series of 1000 artificially generated interferograms with $A=1, B=0$, and $N[t]$ drawn from a Gaussian distribution with a standard deviation of 0.1 (SNR = 10). The root-mean-square (RMS) error of the phase estimation is $0.015 \mathrm{rad}$. We conclude that for this SNR our phase estimation is precise.

Figure 4 suggests, under the assumption that the noise level remains constant in time, that the SNR increases during evaporation. For most regions this statement holds. For some regions, however, the gain in amplitude is reduced by spatial undersampling. Note that the optical spatial resolution $(2 \mathrm{NA} / \lambda)$ is not the limiting factor, but rather the sampling density related to the video camera and the acquisition software. It is important to determine the lowest required SNR for a proper estimation of the phase map. To investigate this empirically, a series of 1000 interferograms were artificially generated with $A=0.01, \ldots, 1.0, B=0$, and $N[t]$ drawn from a Gaussian distribution with a standard deviation of 0.5. For each series the RMS error of the phase estimation is measured. The results of these simulations are shown in Fig. 8. The RMS error of the phase estimation is below 0.16 rad when the SNR is higher than 1. Below this value the RMS error increases rapidly.

\section{Simultaneously Sampling in Space and Time}

In the first part of this section three possible problems that spatial unwrapping algorithms might experience were mentioned. One of these problems is sampling. In this subsection we discuss in detail the aspects of sampling. From a signal-processing point of view there is a fundamental advantage to do the unwrapping in time.

The Nyquist sampling criterion dictates that the fringe pattern needs to be sampled at least at twice the highest frequency in the interferogram to enable reconstruction of the analog fringes. Here, however, we are not interested in reconstruction of the fringes but in reconstruction of the meniscus that produced this fringe pattern. Image acquisition with a CCD camera with a $100 \%$ fill factor for the photosensitive sites, however, is not merely sampling the signal with a two-dimensional impulse train. Image acquisition with a CCD camera can be described in two steps: First, the continuous spatial signal is convolved with the transfer function of the pixel. In the ideal case each pixel on the CCD element has a uniform sensitivity. This implies that the transfer function of a pixel is a block function. Convolution of the signal with the pixel transfer function is equal to a multiplication of the Fourier transform of the signal and the Fourier transform of the pixel transfer function in the frequency domain. The Fourier transform of the pixel transfer function is a sinc function. This sinc function has its first zero crossing at the sampling frequency. The second step in image acquisition is to sample the convolved signal with a twodimensional impulse train. As long as the fringe pattern is spatially sampled at a frequency above the Nyquist frequency, then spatial reconstruction of the fringe pattern is possible with this kind of acquisition.

In our experiments the fringe patterns are sampled in space as well as in time. For this moment we assume that our temporal sampling satisfies the Nyquist criterion. The left image of Fig. 9 shows a one-dimensional fringe pattern generated at the meniscus interface as a function of the contact angle of the meniscus as indicated in Fig. 11, below: From the bottom of this figure upward, the contact angle increases. This results in a steeper meniscus, which is assumed in this example to be straight, and therefore a spatially denser fringe pattern. We assume in this example that the fringe pattern is generated with monochromatic light such that the amplitude of the fringe pattern is constant for different heights. For three different contact angles of the meniscus the amplitude of the fringe pattern is shown. The right image shows the effects of imaging the left image onto a CCD camera. First, the left image is convolved with a uniform filter, the pixel transfer function. As a result of this convolution, the amplitude decreases with increasing frequency, and, at a certain frequency (because of a larger contact angle), the amplitude of the fringe pattern becomes negative. This phase jump of $\pi \mathrm{rad}$ is visualized in region 3 in the right image. Second, the filtered fringe pattern 

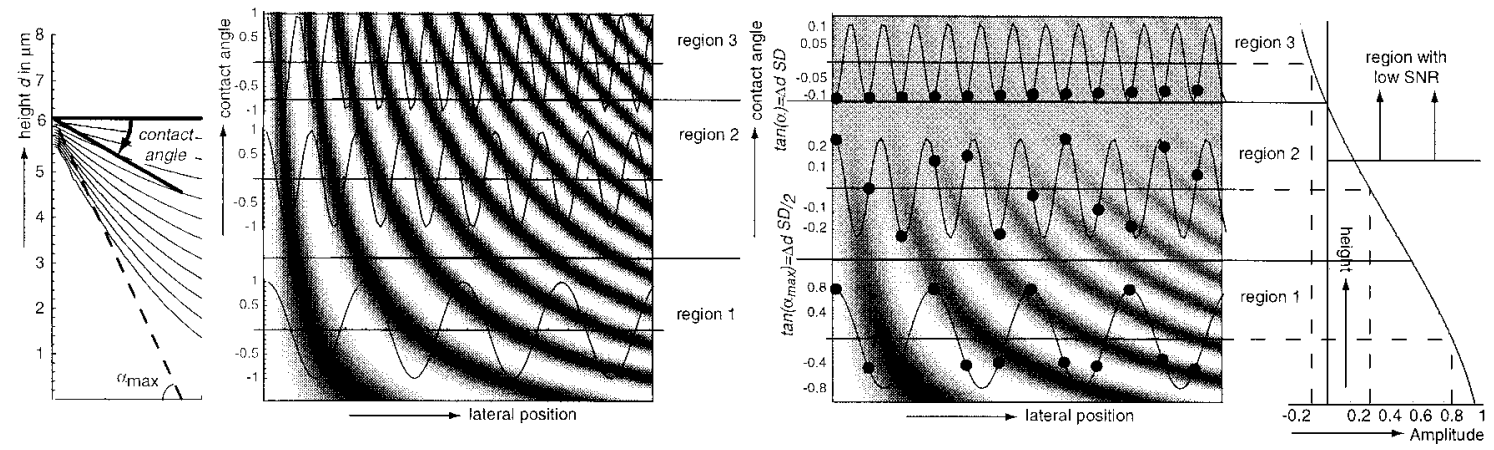

Fig. 9. Left graph shows a fraction of Fig. 11 (below), indicating the contact angle. The left image shows a one-dimensional fringe pattern generated at the meniscus interface as a function of the contact angle of the meniscus as indicated in the left graph: From the bottom upward the contact angle increases, which results in a steeper (straight) meniscus and therefore a spatially denser fringe pattern. We assume in this example that the fringe pattern is generated with monochromatic light such that the amplitude of the fringe pattern is constant for different heights. The right image shows the effects of imaging the left image onto a CCD camera. First, the left image is convolved with a uniform filter, the pixel transfer function. As a result of this convolution, the amplitude decreases with increasing frequency, and, at a certain frequency, the amplitude of the fringe pattern becomes negative. This phase jump of $\pi$ radians is visualized in region 3. Second, the filtered fringe pattern is sampled with a one-dimensional impulse train, indicated by the dots. In region 1 the spatial sampling density SD is larger than the Nyquist frequency $f_{N}$, defined by the contact angle: Spatial as well as temporal unwrapping is possible in this region. In region 2 the spatial sampling density satisfies $f_{N} / 2 \leq \mathrm{SD} \leq f_{N}$ : Aliasing occurs spatially; only temporal unwrapping is possible. In region 3 the spatial sampling density is below $f_{N} / 2$ : No unwrapping is possible, owing to irrecoverable phase jumps.

is sampled with a one-dimensional impulse train, indicated by the dots.

In region 1 the spatial sampling density SD is larger than the spatial Nyquist frequency, defined by the contact angle $\alpha$. Spatial as well as temporal reconstruction of the analog fringe pattern is possible in this region.

In region 2 the spatial sampling density falls below the spatial Nyquist frequency but remains above half the Nyquist frequency. The sinc function corresponding to the pixel transfer function is still positive, as shown in the rightmost part of Fig. 9. This implies that convolution of the signal with the pixel transfer function does not introduce any unrecoverable phase jumps. Each point in the interferogram is spatially undersampled but still sufficiently sampled in time, although with reduced amplitude. This means that the meniscus profile can be reconstructed in time, point by point. Since the meniscus has the smoothest shape possible, this temporal reconstruction also allows for spatial reconstruction of the meniscus. It is, of course, not possible to reconstruct the analog spatial fringe pattern, necessary for spatial unwrapping.

In region 3 the spatial sampling density falls below half the Nyquist frequency. The first negative blob of the sinc function starts to coincide with the Fourier spectrum of the signal, and a phase jump occurs, as shown in the rightmost part of Fig. 9. From this moment on, even temporal reconstruction is not possible.

To summarize, spatial reconstruction of the meniscus requires spatial sampling at the Nyquist frequency, whereas temporal reconstruction requires spatial sampling at half the Nyquist frequency. For this reason we choose temporal reconstruction.

The evaporation speed of the liquid, i.e., the change in height per unit of time, requires a minimal temporal sampling density. Obviously, the evaporation speed is maximal in the center of the vial. The evaporation speed is defined as the height change of a single fringe $\Delta d$ in a time window $\tau_{\Delta d}$. The analysis requires that approximately two periods of the temporal fringe pattern correspond to the fixed window width $K_{w}$. This requirements yields

$$
K_{w}=2 \tau_{\Delta d} R_{\text {sampling }},
$$

where $R_{\text {sampling }}$ is the sampling rate (15 frames/s). According to the Nyquist criterion, this implies 32 times oversampling of the fringe pattern. Note that a wider window implies a better resolution of the discretized frequency spectrum but requires a higher sampling density. Eq. (20) can be rewritten as follows:

$$
v_{\text {evap }}^{\max } \leq \frac{2}{K_{w}} \Delta d R_{\text {sampling }} .
$$

With $K_{w}=128$ and $\Delta d=0.2116 \mu \mathrm{m}$, it follows that $v_{\text {evap. }}^{\max }=0.05 \mu \mathrm{m} / \mathrm{s}$. The evaporation speed in the center of the vial follows from Fig. 4. The average evaporation speed is $6.13 \mu \mathrm{m}$ in $305 \mathrm{~s}$, which equals $0.02 \mu \mathrm{m} / \mathrm{s}$.

However, the spatial sampling density is limited by the angle $\alpha$ between the meniscus and the bottom of the vial: The projection of the fringes on the image sensor becomes denser when the meniscus becomes steeper. The spatial sampling density SD must satisfy the following relation:

$$
\mathrm{SD} \geq \frac{2 \tan (\alpha)}{\Delta d} .
$$


For the interference patterns shown in Fig. 1 the spatial sampling density $\mathrm{SD}=0.91 \mu \mathrm{m}^{-1}$. With $\Delta d=0.2116 \mu \mathrm{m}$ it follows that $\alpha$ must be smaller than 5.5 deg. This criterion will not be met in the regions near the sidewalls of the vial toward the end of the evaporation process. For this reason spatial unwrapping is not possible in these regions. Temporal unwrapping, however, is easy, because the overall change in height in these regions is minimal.

\section{Experiments and Results}

As described in Section 3, the temporal phaseunwrapping algorithm allows for retrieval of the height profile of the meniscus. In this section we show experimental results of these measurements. The measurements are performed on a Zeiss Axioskop with a $20 \times / 0.75$ FLUAR or $5 \times / 0.25$ FLUAR objective. A Sony DXC-960MD 3CCD color video camera is mounted on the microscope with a $2.5 \times$ camera mount from Zeiss. The CCD camera is attached to a Matrox Marval G200 AGP frame grabber. An adjustable cold-light illuminator was used for epiillumination. A 600FS10-50 AM-23831 narrowbandpass interference filter from Andover Corporation was placed directly in front of the illuminator. To direct the light downward in the microscope, a 50/50 beam splitter was placed in the illumination path. Microarrays with different-sized vials were etched in silicon dioxide at the Delft Institute for Microelectronics and Submicron Technology. The vials were manually filled with ethylene glycol with an Eppendorf Transjector 5246. The imageprocessing package SCIL_Image [Organization for Applied Scientific Research (TNO) Institute of Applied Physics (TPD), Delft, The Netherlands] was used to process the video files.

First, the temporal phase-unwrapping algorithm in a single data point as described above was applied to all data points in the region of interest (one quarter of the vial). Figure 10 shows two computed height profiles of the meniscus during evaporation in a 300 $\mu \mathrm{m} \times 300 \mu \mathrm{m}$ vial. Note that the computation of the height profiles is spatially uncorrelated but correlated in time. This correlation in time is inversely proportional to the change of the meniscus level during evaporation. Figure 11 shows the time evolution of a single line of the meniscus during evaporation. The time difference between two successive lines is $10 \mathrm{~s}$. This figure clearly shows that the height change per unit of time is maximal in the center and minimal along the sidewalls of the vials. Furthermore, it shows that the angle $\alpha$ between the meniscus and the bottom of the vial becomes larger than the maximum angle $\alpha_{\max }$ as defined by relation (22). The height profiles in Fig. 11 correspond to the time evolution of the fringes as shown in Fig. 2.

Second, similar measurements are performed in vials with sizes ranging from $75 \mu \mathrm{m} \times 75 \mu \mathrm{m}$ to 300 $\mu \mathrm{m} \times 300 \mu \mathrm{m}$. Table 1 gives a number of important parameters. A straightforward measurement is the remaining liquid volume in the vials as a function of time. Of course, directly related to that is the evap-
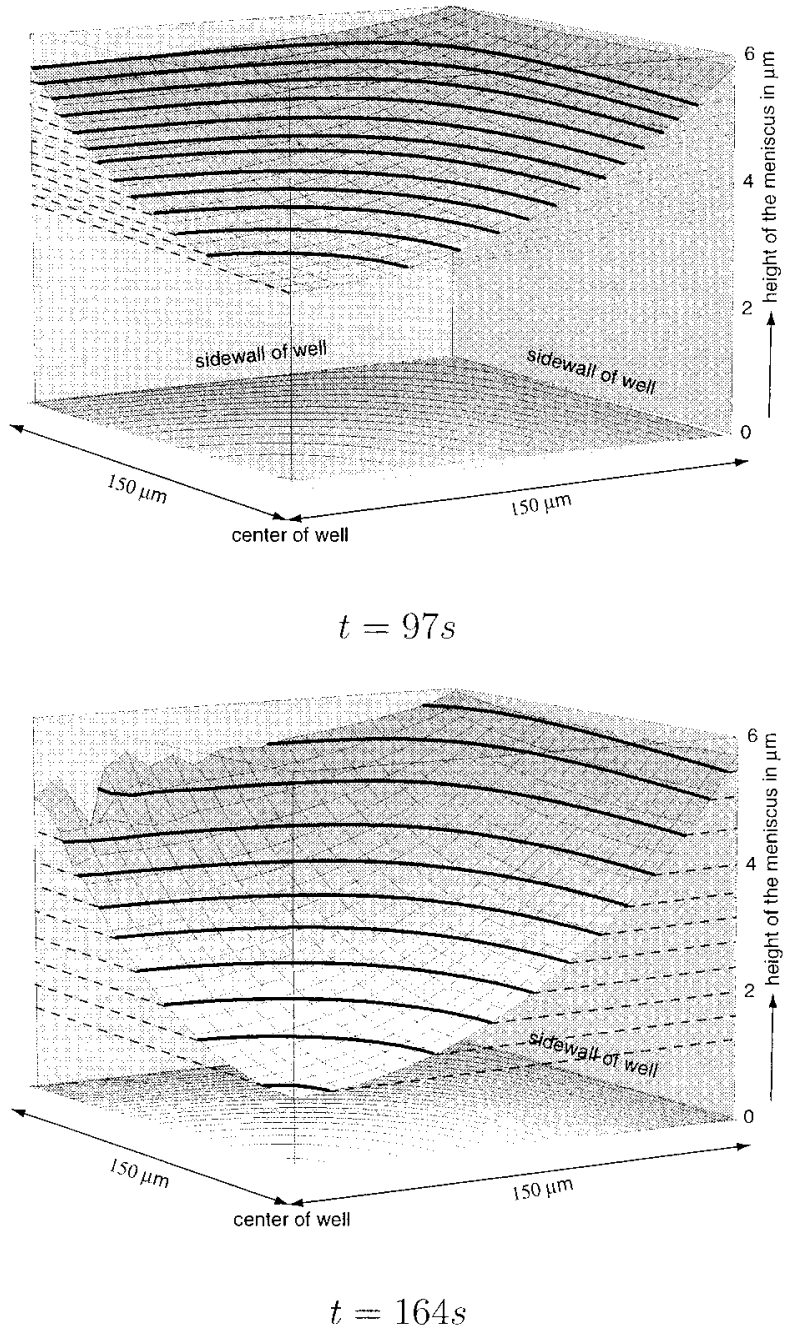

Fig. 10. Computed height profiles corresponding to the interferograms shown in Fig. 1 at $t=97 \mathrm{~s}$ (top) and $t=164 \mathrm{~s}$ (bottom). Because of symmetry only a quarter of the well is shown. Compare the isoheight curves in these figures with the isophote curves in the interferograms. For clarity, only half the number of isoheight curves is shown in the bottom figure.

oration rate of the liquid. The results of these measurements are shown in Fig. 12. The different curves in the top graph of Fig. 12 show how the remaining liquid volumes in the different-sized vials, all with a depth of $6.1 \mu \mathrm{m}$, change as a function of time. The slope of each of these curves is the evaporation rate. The straight lines in this graph indicate that the evaporation rate is constant during evaporation. The evaporation rate as a function of the width of the vial is shown in the bottom graph of Fig. 12. This graph shows that the evaporation rate is proportional to the width of the vial. This is quite a surprising result. Intuitively one would expect the evaporation rate to be proportional to the area of the air-liquid interface, which is roughly proportional to the square of the width of the vial. This counterintuitive result implies that the evaporation process is diffusion limited as explained by Deegan et al. ${ }^{10}$ 


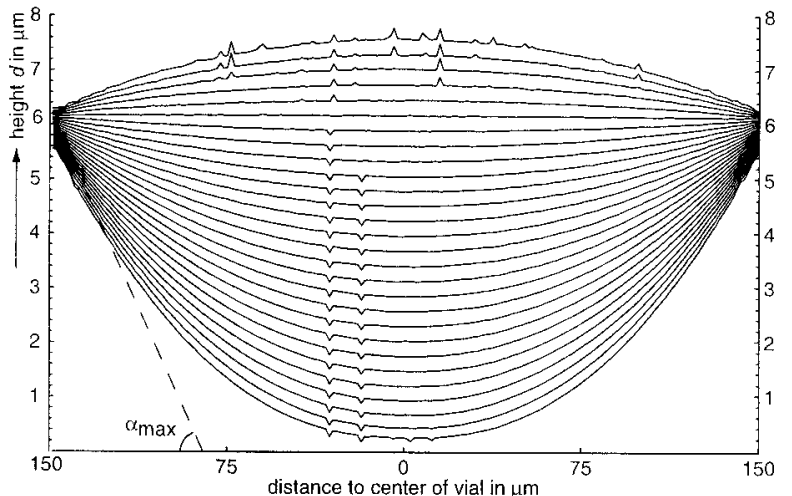

Fig. 11. Time evolution of a single line of the meniscus through the center of the vial. The time difference between two successive lines is $10 \mathrm{~s}$. The angle $\alpha_{\max }$ is the maximum angle between meniscus and bottom for spatial analysis. The glitches in the meniscus are introduced by spurious phase jumps in the unwrapping process.

Third, the temporal phase-unwrapping algorithm is applied to measure height differences in other micromachined picoliter vials. In these specific vials two aluminium electrodes have been put on the bottom of the vial. The electrodes introduce a height difference in the vial, which is observed as a phase jump in the interference pattern. It is, of course, possible to compute only the wrapped phase jump. Therefore the measured absolute height difference limits itself to a maximum of $\Delta d$. The phase jump is computed as follows. First, the interference pattern is recorded, and then the frame where the meniscus is flat is determined. This computation is performed in the region of the vial where the bottom is present. With the algorithm the wrapped phase in each point of this frame is computed. This computation makes the wrapped phase jump $\Delta \Phi$ visible. Because of the different optical behavior of aluminium (phase shift $\pi$ on reflection) and silicon dioxide (no phase shift on reflection), an additional phase difference of size $\pi$ must be added. Since we measure only the wrapped phase jump, an integer number of $2 \pi$ might be added to get the true phase difference $\Delta \Phi^{\prime}$. In formula,

$$
\Delta \Phi^{\prime}=(\Delta \Phi+\pi)+k 2 \pi .
$$
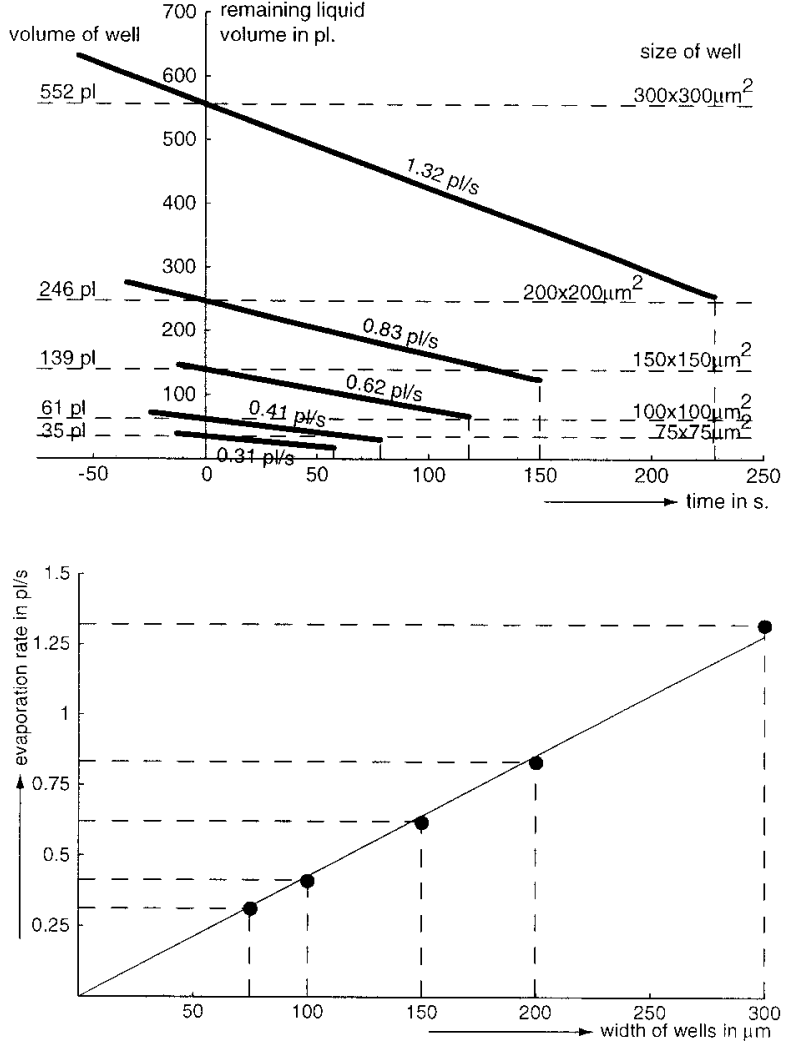

Fig. 12. Upper graph, remaining liquid volume as a function of time for square wells of various size. The depth of the wells is 6.1 $\mu \mathrm{m}$. Bottom graph, evaporation rate as a function of the width of the wells.

We measured the average phase in the computed wrapped phase map in three regions of $25 \times 25$ pixels: the left electrode, the true bottom of the vial, and the right electrode. The average phase of the left electrode is $2.32 \mathrm{rad}$, the average phase of the right electrode is $2.10 \mathrm{rad}$, and the average phase of the bottom is $2.82 \mathrm{rad}$. Given that the electrodes have a defined height of $0.3 \mu \mathrm{m}$, we conclude that the height of the left electrode is $300.6 \mathrm{~nm}$ with a standard deviation of $4.1 \mathrm{~nm}$. The height of the right electrode is $293.2 \mathrm{~nm}$ with a standard deviation of 4.5 $\mathrm{nm}$. The precision on the bottom of the vial is 7.6

Table 1. Measurement Parameters ${ }^{a}$

\begin{tabular}{|c|c|c|c|c|}
\hline Vial Dimensions $\left(\mu \mathrm{m}^{2}\right)$ & Objective & $\begin{array}{l}\text { Region of Interest } \\
\text { (pixels) }\end{array}$ & $R_{\text {sampling }}\left(\mathrm{s}^{-1}\right)$ & Frames (flat/total) \\
\hline $75 \times 75$ & $20 \times / 0.75$ & $175 \times 155$ & 15 & $193 / 1055$ \\
\hline $100 \times 100$ & $20 \times / 0.75$ & $113 \times 206$ & 15 & $368 / 1544$ \\
\hline $150 \times 150$ & $20 \times / 0.75$ & $173 \times 159$ & 30 & $362 / 3906$ \\
\hline $200 \times 200$ & $5 \times / 0.25$ & $118 \times 106$ & 15 & $528 / 2776$ \\
\hline $300 \times 300$ & $20 \times / 0.75^{b}$ & $136 \times 258$ & 15 & $1040 / 4572$ \\
\hline
\end{tabular}

${ }^{a}$ First column, dimensions of the different vials. All vials have a depth of $6.1 \mu \mathrm{m}$. Second column, objective used for acquisition. For all but one vial, a $2.5 \times$ camera mount was used. Third column, region of interest of each vial. For reasons of symmetry this region is only a quarter of the vial. Fourth column, temporal sampling rate. Fifth column, the length of the recording and the frame where the meniscus is flat.

${ }^{b} 1.0 \times$ Camera mount used instead of $2.5 \times$ camera mount. 

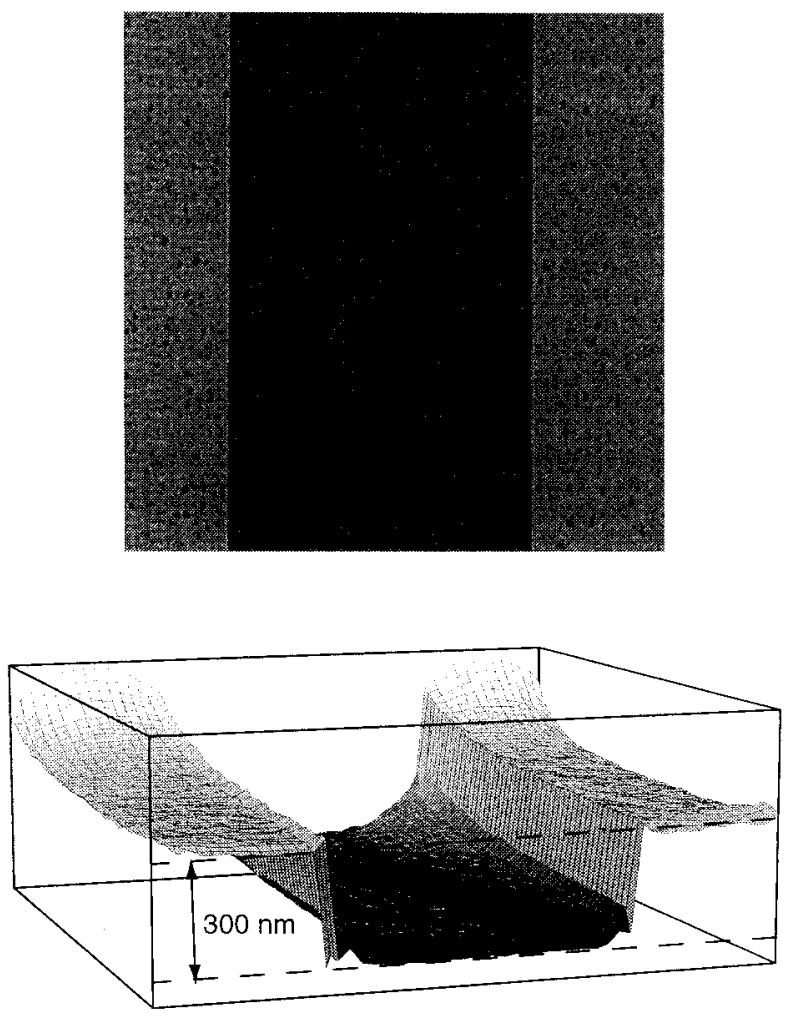

Fig. 13. Upper image, electrodes patterned on the bottom on both sides of the vial. Bottom figure, measured height difference between the bottom of the vial and the electrodes.

$\mathrm{nm}$. The final result of this computation is shown in Fig. 13.

\section{Conclusions and Discussion}

A study of the dynamic behavior of liquid evaporation in subnanoliter vials requires that the liquid volume be properly sampled in time as well as in space. Video microscopy allows for sufficient sampling in time but lacks axial resolution. In contrast, confocal fluorescence microscopy has sufficient axial resolution but at the expense of temporal resolution. Interference-contrast microscopy complies with both sampling requirements. With this type of microscopy, fringe patterns in the liquid are observed showing fringes of equal height.

First, this paper presented the underlying optical model of this type of microscopy. This model is based on the classical theory of the generation of fringes of equal thickness in thin films. This model is extended by means of taking polarization, different angles of incidence, and the spectrum of the incident light into account. The simulation results based on numerical evaluation of the optical model are compatible with our experimental results. There is, however, a rather poor agreement between the observed and the theoretical fringe visibility. This is due to out-of-focus light. The error between the simulated positions of the zero crossings of the modulations and the measured positions shows a standard deviation of $2.5 \mathrm{~nm}$. From the simulation results we measured that for our experimental setup the length of the modulations equals $211.6 \mathrm{~nm}$.

Second, this paper introduced a phase-unwrapping algorithm. The unwrapping is performed as a function of time for a fundamental reason. A spatial unwrapping algorithm requires spatial sampling at a spatial frequency at least as high as twice the highest frequency in the interferogram, to meet the requirements of the Nyquist sampling theorem. In this paper we have explained that temporal unwrapping requires a spatial sampling frequency of at least half the Nyquist sampling frequency for CCD sensors with $100 \%$ fill factor. Because of the continuum of phase differences between two successive instants in time, we propose using a window function with a variable width in terms of physical units (fixed width in terms of data points). This requires subsampling of the interferogram to obtain approximately two periods of the fringe pattern within the size of the window. The phase estimation in our algorithm has a bias less than $0.01 \mathrm{rad}$. A typical SNR in our measurements is 10 . With this SNR the RMS error of our phase estimation is $0.015 \mathrm{rad}$. This corresponds to an axial resolution of $0.5 \mathrm{~nm}$. The minimum SNR for a proper phase estimation is 1 . A key point in the algorithm is that all measurements are spatially uncorrelated. Furthermore, a valid reference point can be computed to calibrate all relative heights. This allows for absolute height measurements. We have successfully applied this algorithm to monitor the evaporation process in different-sized vials. A striking result is that the evaporation rate depends linearly on the width of the vial. This algorithm can also be applied to measure height differences of objects on the bottom of a vial. We have shown that height differences can be measured with a precision of approximately $5 \mathrm{~nm}$. This axial resolution is at least two orders of magnitude better than the lateral resolution. We are convinced that this algorithm is applicable in other types of measurements in which dynamic interference patterns are observed.

The authors gratefully acknowledge the reviewers for their detailed comments on our manuscript. This research was supported by the Delft Interfaculty Research Center Intelligent Molecular Diagnostic Systems.

\section{References}

1. K. T. Hjelt, G. W. Lubking, M. J. Vellekoop, L. J. van Vliet, L. R. van den Doel, A. Greiner, and I. G. Korvink, "Nanoliter droplet behavior in micromachined wells," in Sensors Update, 1st ed., H. Baltes, W. Gopel, and J. Hesse, eds. (Wiley-VCH, Weinheim, Germany, 2000), Vol. 8, pp. 39-72.

2. M. Born and E. Wolf, Principles of Optics, 6th ed. (Pergamon, Oxford, UK, 1980).

3. A. Lambacher and P. Fromherz, "Fluorescence interferencecontrast microscopy on oxidized silicon using a monomolecular dye layer," Appl. Phys. 63, 207-216 (1996).

4. S. Inoué, Video Microscopy (Plenum, New York, 1986).

5. L. Mertz, Transformations in Optics (Wiley, New York, 1965). 
6. M. Servin, F. J. Cuevas, D. Malacara, J. L. Marroquin, and R. Rodriquez-Vera, "Phase unwrapping through demodulation by use of the regularized phase-tracking technique," Appl. Opt. 38, 1934-1941 (1999).

7. J. Strand, T. Taxt, and A. K. Jain, "Two-dimensional phase unwrapping using a block least-squares method," IEEE Trans. Image Process. 8, 375-386 (1999).

8. J. M. Huntley and H. Saldner, "Temporal phase unwrapping for automated interferogram analysis,” Appl. Opt. 32, 30473052 (1993).

9. A. V. Oppenheim, A. S. Willsky, and S. H. Nawab, Problem 3.66. in Signals and Systems (Prentice-Hall, New Jersey, 1997), p. 275.

10. R. D. Deegan, O. Bakajin, T. F. Dupont, G. Huber, S. R. Nagel, and T. A. Witten, "Contact line deposits in an evaporating drop,” Phys. Rev. E Part B 62, 756-765 (2000). 